\title{
Los recursos didácticos tecnológicos en el desarrollo de las habilidades lectoras en los estudiantes de Educación Básica
}

Technological didactic resources in the development of reading skills of basic education students.

Paulina del Roció Pullupaxi Pullupaxi. ${ }^{1}$, Lourdes Elizabeth Navas Franco. ${ }^{2}$ Jhon Patricio Acosta Bonilla. ${ }^{3} \&$ Alexandra Wilma Paredes Guevara. ${ }^{4}$

\begin{abstract}
.
DOI: https://doi.org/10.33262/cienciadigital.v3i2.6.518

The constant change that causes the use of technological didactic resources in the educational field becomes transcendental, current education requires appropriate didactic processes that achieve significant learning and the development of competences. The objective is to facilitate the acquisition of reading skills by using technological resources to promote the students' competitive progress. To do this, wit followed a mixed methodology: qualitative-quantitative which allowed to check review the regulations, curriculum and the research background. In the field study, a test was carried out to identify reading skills in the students, and an interview to the teachers of the institution allowing data collection and their respective analysis. According to the gotten results through the analysis and interpretation, it determines a limited understanding by the reader, giving a proposal to the use of technological didactic resources which will improve that problem in the children of third grade of "La Granja" elementary school and it will also allow the development of fluent reading by the use of teaching resources. Among the main conclusions, it is significant to mention that the implementation of informative and technological didactic resources will awaken the interest, motivation, imagination, creativity and ability in the students to solve problems according to their age.
\end{abstract}

Keywords: Methodology, reading skills, technological didactic resources.

\footnotetext{
1 Universidad Tecnológica Indoamérica, Maestría en Educación, mención Innovación y Liderazgo Educativo. Ambato, Ecuador. paulina_pullupaxi@yahoo.es

${ }^{2}$ Universidad Tecnológica Indoamérica, Facultad de Ciencias Humanas, de la Educación y desarrollo social, Carrera de Educación Básica e Inicial. Ambato, Ecuador. lourdesnavas@uti.edu.ec

${ }^{3}$ Universidad Tecnológica Indoamérica, Facultad de Ciencias Humanas, de la Educación y desarrollo social, Carrera de Educación Básica e Inicial. Ambato, Ecuador. jhonacosta@uti.edu.ec

${ }^{4}$ Universidad Tecnológica Indoamérica, Facultad de Ciencias Humanas, de la Educación y desarrollo social, Carrera de Educación Básica e Inicial. Ambato, Ecuador. alexandraparedes@uti.edu.ec
} 


\section{Resumen.}

El cambio constante que provoca el uso de los recursos didácticos tecnológicos en el ámbito educativo se torna transcendental, la educación actual requiere de procesos didácticos adecuados que logren un aprendizaje significativo y el desarrollo de competencias. El objetivo es facilitar la adquisición de habilidades lectoras mediante recursos tecnológicos para favorecer el progreso competitivo de los estudiantes. Para ello se siguió una metodología mixta cualitativa-cuantitativa que permitió la revisión bibliográfica de las normativas, currículo y antecedentes investigativos; en el estudio de campo se efectuó un test para identificar habilidades lectoras en los estudiantes, y una entrevista a los docentes de la institución permitiendo así la recolección de datos y su respectivo análisis. En función a los resultados obtenidos mediante el análisis e interpretación determina una limitada comprensión lectora, dando como propuesta el uso de recursos didácticos tecnológicos que mejoraren dicho problema en los niños de tercer grado de la escuela de educación básica "La Granja", el cual permitirá desarrollar fluidez lectora mediante el uso de los recursos didácticos. Dentro de las principales conclusiones se destaca que el implementar los recursos tecnológicos digitales y de información despertará en los estudiantes el interés, motivación, imaginación, creatividad y capacidad para solucionar problemas acordes a su edad.

Palabras claves: Destreza, habilidades lectoras, metodología, recursos didácticos tecnológicos.

\section{Introducción.}

Las instituciones educativas en la actualidad sienten la necesidad de incorporar el uso y aplicación de los recursos digitales TICS, dado el cambio constante que provoca el uso de las mismas dentro del contorno educativo se vuelve importante estudiarlo e incorporarlo al desarrollo de los niños dentro de las habilidades lectoras.

El cual se muestra como un tema de actualidad porque ofrece herramientas de enseñanzaaprendizaje en todas las áreas de la educación, debido a que la sociedad actual está fundamentada en el conocimiento científico y tecnológico que producen cambios acelerados, manteniéndose como un motor de la colectividad que busca desarrollar las capacidades físicas, intelectuales y morales del ser humano para posibilitar la adquisición de saberes y mejorar progresivamente la calidad de vida.

Las habilidades lectoras se amparan bajo la teoría de Vygotsky quien sostenía que los niños/ñas desarrollan su aprendizaje por la interacción social, que permite adquirir nuevas y mejores habilidades cognoscitivas como un proceso lógico mientras van interactuando en cada etapa de su vida. Según Vygotski (1929) y citado por (Rodríguez \& Maldonado, 2017) manifiestan que el conocimiento de la persona se produce desde un plano interpersonal hacia un plano intrapersonal, debido a que el ser humano nace en un contexto sociocultural pre-existente y, por tanto, enfrenta el imperativo de internalizarse 
poco a poco en las actividades culturales, hasta que las mismas se transformen en actividades mentales.

Para (Alaís \& Sarmiento, 2014) establecen que proceso educativo es un proceso de andamiaje, es decir, las ayudas, interacciones y relaciones que se establecen entre un profesor experto, un alumno aprendiz y un texto contenido o material de aprendizaje", (p. 1033), bajo esta perspectiva, aprender y enseñar reside en mantener espacios para el conocimiento compartido en el que un experto ayuda a un aprendiz a apropiarse del uso de un determinado material de aprendizaje, por último, las funciones mentales en desarrollo deben ser evaluadas mediante actividades cooperativas o situaciones.

Las habilidades lectoras toman diferentes sinónimos conforme se lo indica a continuación, el modelo de evaluación de PISA (2008) y citado por (Castillo, 2017) se centra en el concepto de literacy como "aptitud o competencia, aunque en diferentes países ha sido traducido como cultura, formación, alfabetización o habilidad que los estudiantes deben desarrollar" (p. 2). En Ecuador las habilidades lectoras hacen referencia a las competencias lectoras. Partiendo de forma general una competencia es "pericia, aptitud, o idoneidad para hacer algo o intervenir en un asunto determinado.

La lectura infantil es una etapa fundamental de todo niño (es la fase donde se presenta el reconocimiento de letras, adquiriendo el código oral y escrito, siguiéndole la conciencia fonológica), siendo importante porque en esta etapa es donde aprenderán a desarrollar los hábitos lectores o simplemente a desinteresarse, debiendo los padres, tutores y maestros influenciar en ellos la lectura.

Para (Ríos \& Cardona, 2016) el aprendizaje de la lectura debe tener relación con el aprendizaje del código escrito, en los niños en la etapa preescolar es necesario los elementos como la rima y la aliteración, el conocimiento de los fonemas y su relación con los grafemas, es decir, para el reconocimiento de palabras en representación escrita, amerita realizar una conversión de la ortografía en sonidos, examinar la estructura sonora del habla y reconocer que está en un sistema escrito.

Recordar hechos tal y como aparecen en el texto. Para (Tierra, 2016) "este nivel se refiere a que el lector reconoce las frases y palabras claves del contenido. Y es más factible la comprensión del texto ya que comprende el reconocimiento de la estructura del texto" (p. 17).

Es la atribución de significados que son relacionados con el conocimiento previo. Según Gordillo (2007) y citado por (Tierra, 2016) este nivel se determina en relacionar y asociar significados ya que admiten al lector leer entre líneas, "presuponer y deducir lo implícito; es decir, busca relacionar el texto más allá de lo leído, expone el texto con amplitud y agrega sus conocimientos anteriores, relaciona el texto, los conocimientos previos, formulando hipótesis y nuevas ideas" (p. 17).

El nivel de comprensión crítica se refiere a la emisión de juicios de valor. Según Cassany (2006) citado por (Serna, 2015) "la comprensión crítica busca lo que está detrás de las 
líneas, lo que hay detrás de las líneas es la ideología, el punto de vista, la intención y la argumentación que apunta realizar el autor" (p. 173).

Sí, se perfecciona el uso eficiente de los niveles de las habilidades lectoras el estudiante logrará mejorar la habilidad de leer y emitir juicios de valor, además permitirá elaborar el significado de las palabras por la vía de aprender las ideas relevantes del contenido del texto y relacionarlo con las ideas que ya se tienen. Para los niños y niñas parten de lo que conocen a obtener y ampliar la experiencia sobre el mundo y sus formas de representación y comunicación a través de la lectura, siendo la escuela la encargada de ofrecerles la oportunidad de asimilar la modalidad más neutra de representación de la lengua verbal y escrita.

Según (Gusqui \& Tixi, 2017) determinan que "es el desarrollo de significados a través de la adquisición de ideas más significativas de un texto permitiéndole establecer vínculos entre las ideas adquiridas con anterioridad" (p. 32), acoplando a esta definición, es la habilidad que tiene un individuo para comprender el lenguaje escrito, partiendo de la decodificación del texto hasta llegar a un diálogo entre el contexto del autor y los conocimientos previos del lector, creando nuevos conocimientos.

La velocidad lectora es la capacidad que se tiene de leer palabras en un determinado lapso de tiempo tratando de comprender lo leído. (Gusqui \& Tixi, 2017) manifiestan que "como primer proceso consiste en captar una información y mandarla al cerebro en un determinado lapso de tiempo, en el que se produce el segundo proceso, el comprensivo que es codificando un sistema de señales e interpretar símbolos abstractos” (p. 32). Hay que tomar en cuenta que leer en voz alta ayuda a la concentración y a mayor velocidad disminuye la oportunidad de perder la concentración mental porque la capacidad de interpretar lo escrito es más veloz que la habilidad de pronunciar palabras mientras se lee.

(Gusqui \& Tixi, 2017) argumenta que la fluidez lectora para "es la capacidad de leer rápido, suave y con entonación, el lector debe entender como los símbolos se relacionen con los sonidos de la lengua para formar palabras" (p. 32). Considerada como la habilidad para leer en voz alta con entonación, ritmo, fraseo y pausas adecuadas para que la lectura sea entendida y con sentido, al identificar las ideas enmarcadas por la puntuación.

Las estrategias lectoras se interpretan como la selección y uso de procedimientos de aprendizaje que permiten una lectura activa, intencional, autorregulada y competente en función del objetivo planteado y las características del texto.

Para Bereiter y Scardamalia (1989); Paris y Paris, (2007); Paris, Lipson y Wixson (1983) citado por (Gutierrez \& Salmeron, 2012) "las estrategias lectoras hacen referencia a las habilidades dirigidas a la consecución de una meta, estas se clasifican en: cognitivas, metacognitivas, motivacionales-afectivas, y contextuales" (p. 185).

Fierro (2011) manifiesta que las estrategias cognitivas "se trata de un enfoque sobre lo mental, constituido por un conjunto de conocimientos con principios básicos, problemas 
y soluciones" (p. 520). En tal sentido las estrategias lectoras cognitivas obedecen a procesos dinámicos y constructivos de la mente que el lector realiza de forma consciente e intencional para construir en la mente el texto escrito.

Para Graesser (2007) y Williams y Atkins (2009) citado por (López, Valladares, \& otros, 2014) las estrategias Metacongnitivas "implica un proceso consciente donde el estudiante en su papel de lector debe ser capaz detectar y solucionar los problemas de comprensión generados en la lectura de un determinado texto" (2014).

(Muñoz \& Ocaña, 2017) mencionan que se puede establecer tres etapas dentro de las estrategias meta cognitivas fundamentales para la comprensión: antes de iniciar la lectura she debe ayudar al estudiante en el proceso de comprensión a través de estrategias, por ejemplo, revisando y actualizando el conocimiento previo, porque la información o conocimiento previo es un componente fundamental de comprensión lectora antes de leer cualquier texto; durante la lectura se debe realizar la evaluación que involucra elaborar resúmenes y esquemas que den cuenta del texto, analizar errores cometidos y planear soluciones; y después de la lectura se debe corregir los errores cometidos, elaborar una representación integral y propia del texto y extender el conocimiento que se obtuvo de la lectura.

\section{Metodología.}

La investigación es de carácter descriptivo, con un enfoque mixto, no experimental, que caracteriza los recursos didácticos tecnológicos para mejorar las habilidades lectoras. La necesidad de aplicar recursos didácticos tecnológicos para mejorar las habilidades lectoras en los estudiantes de tercer año de educación general básica de la Unidad Educativa "La Granja", de la ciudad de Ambato en Ecuador, radica en que la Institución requiere validar nuevas estrategias de enseñanza que habiliten a los niños a fortalecer sus competencias y habilidades desde temprana edad.

Se aplicó el Test de Habilidades Lectora (Pascual, 2018) que permite medir las diversas facultades intelectuales del individuo lo que permite realizar una clasificación. quienes determinaron que evalúa los procesos cognitivos, consientes y deliberados que deben reconocer un test de comprensión lectora.

El test parte de la lectura "la rana que quería ser una rana auténtica" que complementa la aplicación del mismo, está compuesto de 10 ítems, cada uno con tres opciones de respuestas. Esta técnica permitió recolectar información clara y precisa, en cuanto al nivel de habilidades lectoras de los niños de la institución, para poder analizar e interpretar los datos que nos proporcionen los estudiantes.

Se aplicó una entrevista dirigida a los docentes y autoridades de la institución en la que se determinó el uso de los recursos didácticos tecnológicos en el desarrollo de las habilidades lectoras de los estudiantes a través de preguntas abiertas. 
Para el procesamiento de la información de esta investigación se trabajó con una muestra de 23 estudiantes de la Escuela de Educación Básica "La Granja", donde se aplicó la técnica del test en el que respondieron 10 preguntas. Para realizar el diagnóstico inicial de la problemática, se apeló al uso de tabulación de datos, diseño de cuadros y gráficos porcentuales, en donde se implantó el análisis e interpretación de resultados mediante el programa estadístico SPSS.

Por ser estudiantes de tercer grado se requirió el apoyo de los docentes para la explicación de los objetivos y procedimientos de estudio, Se solicitó la autorización de los padres de familia y de las autoridades de la institución para diagnosticar la realidad existente las habilidades lectoras y el uso de la tecnología en los procesos pedagógicos desarrollados en la institución.

\section{Resultados.}

Los resultados obtenidos de la aplicación del test de habilidades lectoras dirigido a los estudiantes de la Escuela de Educación Básica "La Granja" se obtuvieron a partir de la lectura "La Rana que quería ser una rana auténtica" de Augusto Monterroso, a través de esta lectura los estudiantes fueron contestando el test de diez preguntas que tienen opciones de respuesta en base a lo leído.

Los estudiantes evaluados muestran gran deficiencia en habilidades lectoras ya que no logran identificar correctamente el tipo de texto leído y con dificultad reconocen a sus protagonistas; las ideas más importantes descritas por los estudiantes no son significativas y no aportan al desarrollo de la actividad; en la mayoría de los casos no logran identificar las características de la lectura. Los resultados se resumen a continuación:

Tabla 1 Resultados del test de habilidades lectora

\begin{tabular}{lll}
\hline Alternativas & Frecuencia & Porcentaje \\
\hline 1.- De estas posibilidades, elige la que creas correcta: & \multicolumn{2}{l}{} \\
Lo que acabo de leer es un texto narrativo & 5 & 21,7 \\
Se trata de un texto de tipo descriptivo con algo de narración & 6 & 26,1 \\
Es un texto de lírica popular & 12 & 52,2 \\
2. Los textos en que los protagonistas son animales se llaman: & \\
Narraciones épicas & 6 & 26,1 \\
Fábulas & 12 & 52,2 \\
Odas & 5 & 21,7 \\
3. La historia que acabas de leer es: & & \\
Un cuento de literatura popular & 12 & 52,2 \\
Un cuento literario de un autor culto. & 6 & 26,1 \\
Un texto no literario & 5 & 21,7 \\
\hline
\end{tabular}




\begin{tabular}{|c|c|c|}
\hline \multicolumn{3}{|c|}{$\begin{array}{l}\text { 4. Como casi todos los textos de este tipo, el que has leído } \\
\text { consta de las siguientes partes: }\end{array}$} \\
\hline Introducción-desarrollo-conclusiones. & 6 & 26,1 \\
\hline Presentación del tema-argumentos-recapitulación final & 5 & 21,7 \\
\hline Planteamiento-nudo-desenlace & 12 & 52,2 \\
\hline \multicolumn{3}{|c|}{$\begin{array}{l}\text { 5. Para intentar ser lo más "auténtica" posible, lo primero que } \\
\text { hizo la rana fue: }\end{array}$} \\
\hline Buscarse un ansioso espejo muy largo. & 6 & 26,1 \\
\hline Comprarse un espejo donde mirarse largo rato & 12 & 52,2 \\
\hline Esforzarse mucho en conseguirlo & 5 & 21,7 \\
\hline \multicolumn{3}{|l|}{ 6. La rana de la historia encuentra su autenticidad... } \\
\hline Según el humor del día o de la hora & 7 & 30,4 \\
\hline Según la opinión de los demás & 12 & 52,2 \\
\hline Dependiendo de lo que veía en el espejo & 4 & 17,4 \\
\hline \multicolumn{3}{|l|}{ 7. Para conocer su verdadero valor, la rana... } \\
\hline Guardó el espejo en un baúl & 5 & 21,7 \\
\hline Decide fijarse en la opinión de los demás. & 12 & 52,2 \\
\hline Cambió de peluquería & 6 & 26,1 \\
\hline \multicolumn{3}{|l|}{ 8. Los demás admiran de la rana: } \\
\hline El sabor de sus ancas. & 5 & 21,7 \\
\hline Su forma de hacer sentadillas. & 6 & 26,1 \\
\hline La belleza de sus piernas. & 12 & 52,2 \\
\hline
\end{tabular}

9. De las siguientes palabras del texto, señala la que sea derivada:

Autenticidad

Humor

10. Los conectores son las palabras o grupos de palabras que sirven para relacionar unas ideas o acciones con las otras, siguiendo un orden lógico. Señala cuál es el orden lógico de los conectores en la historia que acabas de leer:

Había una vez - al principio - por fin

Al principio - luego - finalmente.

1252,2

Había una vez - un día - al principio

$7 \quad 30,4$

TOTAL

$7,76 \quad 33,33$

Resultados obtenidos de la aplicación del test de habilidades lectoras dirigido a los niños de la Escuela de Educación Básica "la Granja" que se utilizó para determinar el problema planteado.

Figura 1. Resumen de la aplicación delTest de habilidades lectoras realizada a los estudiantes de Educación Básica de la escuela "La Granja"" 


\section{Resumen Test de habilidades lectoras}

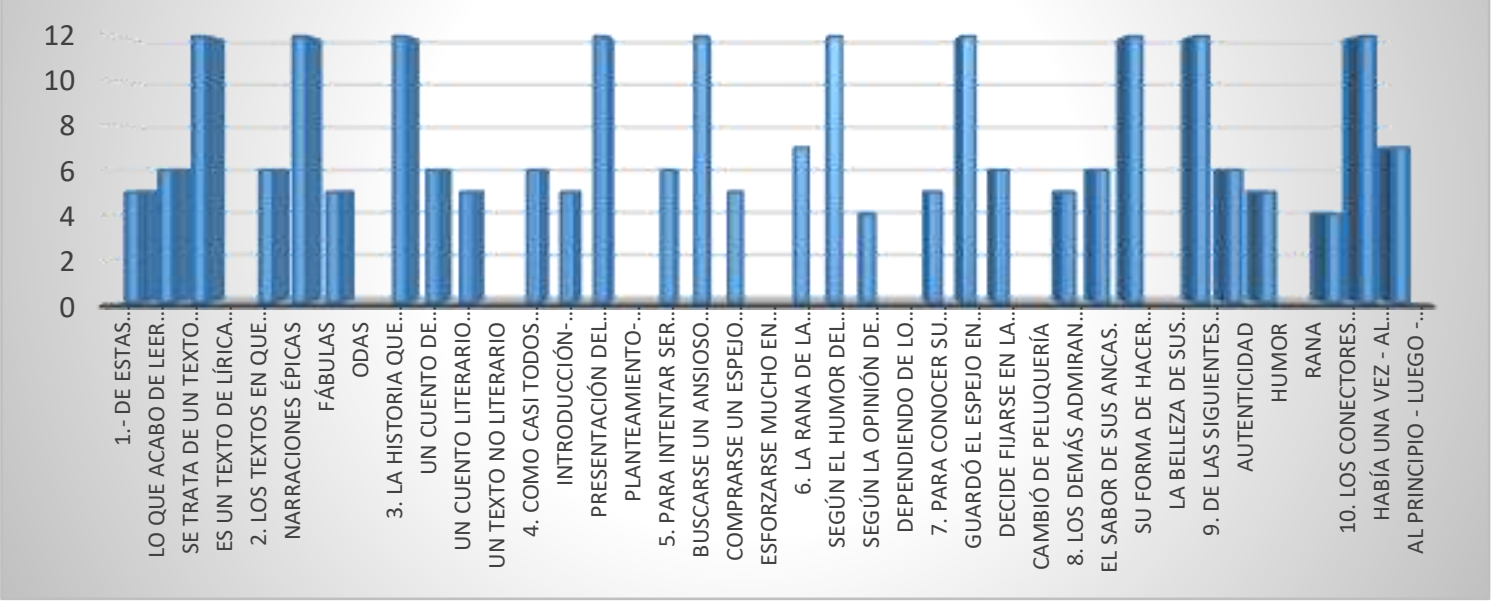

Después de algunos días se aplicó el test nuevamente, pero con la ayuda de un recurso tecnológico y los resultados fueron positivos ya que los estudiantes identificaron claramente el tipo de texto, identificaron a los protagonistas del texto, reconocieron las partes del texto, se observó claramente el valor a desarrollar, es decir los estudiantes lograron formular varias hipótesis, la verificaron y lograron la integración de la información, controlando su comprensión lectora de forma satisfactoria.

Se realizó la entrevista dirigida a los docentes de la Escuela de Educación Básica "La Granja" en donde se observó el siguiente resultado:

\section{Pregunta 1. ¿Conoce qué son recursos didácticos digitales?}

Existe un conocimiento básico de los recursos didácticos digitales, puesto que los docentes creen que son computadoras, páginas web y todo objeto electrónico y digital que sirve como técnica dentro del proceso educativo. Ciertamente los recursos didácticos digitales son aquellos que se encuentran en la web y son creados con fines educativos y su diseño responde a unas características didácticas apropiadas para la enseñanzaaprendizaje de los usuarios.

\section{Pregunta 2. ¿Conoce para qué sirven los recursos didácticos digitales?}

En referencia al uso de los recursos didácticos digitales la docente cree que es para apoyar en el proceso de enseñanza- aprendizaje, más no para que por sí solos encaminen a los niños hacia el conocimiento. Así mismo, se puede acoplar que estos con el uso adecuado permiten llamar la atención y motivar a los estudiantes con nuevas estrategias porque constituyen nuevas formas de representación, enriquecida con imagen, sonido y video digital que requiere de un computador, un dispositivo móvil y conexión a Internet. 
Pregunta 3. ¿Utiliza para el desarrollo de la clase algún dispositivo electrónico? ¿Cuáles?

La docente no utiliza durante la clase ningún tipo de dispositivo electrónico, porque el aula no está equipada y en el caso de querer utilizar el laboratorio de computación debe solicitarlo con mucho tiempo de antelación, así que el proceso de enseñanza lectora lo realiza generalmente con técnicas tradicionales o aquellas que no sean con dispositivos electrónicos.

Pregunta 4. ¿Aplica durante la clase recursos didácticos tecnológicos para el desarrollo de la comprensión lectora? ¿Cuáles?

Durante la clase no aplica recursos didácticos tecnológicos para el desarrollo de la comprensión lectora, por los motivos antes mencionados, pero sí envía a veces consultas a la casa para que los niños con ayuda de los padres puedan realizar una lectura digital, como por ejemplo la lectura digital de un artículo en la Revista "La pandilla", pero no es tan fiable puesto que los padres prefieren comprar la revista y dejar que el niño lea solo.

Pregunta 5. ¿Cree que le hace falta de capacitación en recursos didácticos tecnológicos para el desarrollo de la comprensión lectora?

La docente está muy consiente que le falta capacitación en el uso de recursos didácticos tecnológicos para el desarrollo de la comprensión lectora, dice que le encantaría motivar a sus estudiantes en el aprendizaje de la lectura ofreciéndoles nuevas formas de presentación, formatos animados con actividades que ayuden a desarrollar los hábitos lectores en los niños, videos y material audiovisual.

\section{Discusión.}

Se plantea una aplicación de recursos didácticos tecnológicos para mejorar las habilidades lectoras en los estudiantes de tercer grado de la Escuela de Educación Básica "La Granja. En donde se pueda desarrollar la comprensión lectora con el uso de las TICS, es decir, el uso de dispositivos electrónicos, procesadores de texto básicos y la web, ingresándole al niño a un nuevo mundo en la lectura, la "lectura digitalizada" donde podrá hacer uso de nuevas herramientas que generen su curiosidad y a la vez diversión, preparándole académicamente para manejar dichas herramientas de forma responsable y con necesidades educativas que favorezcan a su aprendizaje.

Para el desarrollo de recursos didácticos tecnológicos que mejoren las habilidades lectoras se han establecido en tres fases para su estructura y uso: la fase uno para el diseño de recursos didácticos tecnológicos se han establecido dos procesos, la segunda fase es la estructura de las actividades planteadas con el recurso tecnológico y; la tercera fase que es la aplicación de estas actividades.

Los recursos didácticos tecnológicos se han establecido como herramientas para el docente de Lengua y Literatura, que le permitirá desarrollar la comprensión lectora en los alumnos de tercer año de básica con el uso de las TICS, es decir, el uso de dispositivos 
electrónicos, procesadores de texto básicos y la web, ingresándole al niño a un nuevo mundo en la lectura, la "lectura digitalizada" donde podrá hacer uso de nuevas herramientas que generen su curiosidad y a la vez diversión, preparándole académicamente para manejar dichas herramientas de forma responsable y con necesidades educativas que favorezcan a su aprendizaje.

Tomando en cuenta que son niños que cuentan con conocimientos empíricos de las TICS y se encuentran en el proceso de aprendizaje de la lectura y escritura se realizará actividades básicas, utilizando en la mayor parte de las actividades el "mouse". (Gutiérrez, 2013) las tecnologías de la información y la comunicación (TIC) se perfilan como una de las competencias básicas que los docentes han de haber adquirido para el desempeño de su profesión.

La incorporación de los Recursos Didácticos Tecnológicos, a la educación se ha transformado en un proceso, cuya implicación, va mucho más allá de las herramientas tecnológicas que conforman el contexto educativo, "se habla de una construcción didáctica y la manera cómo se pueda construir y consolidar un aprendizaje significativo en base a la tecnología, en estricto pedagógico se habla del uso tecnológico a la educación" (Hernandez, 2017).

Para Elosúa (2014) las habilidades lectoras son una herramienta primordial para la comprensión de textos y el beneficio académico. El papel de la comprensión en el transcurso de escolarización, fundamentalmente en la fase de Primaria, ha sido acentuado por algunos investigadores. Existen destrezas lectoras que tienen que ver más con los métodos de descodificación (por ejemplo, la segmentación de palabras), mientras que otras habilidades involucrarían procesos de comprensión propiamente (por ejemplo, la realización de inferencias). A medida que se van adelantando las primeras, se van desplegando también las segundas, ya que se liberan recursos cognitivos que pueden dedicarse a la comprensión.

\section{Conclusiones.}

- Es fundamental el detectar el nivel de desarrollo de habilidades lectoras en los estudiantes de tercer grado de la Escuela de Educación Básica "La Granja". Mediante un test realizado a los estudiantes se determinó que la mitad de la población presenta problemas en la comprensión lectora de un texto, evidenciándose de esta forma que se necesita la implementación de nuevas estrategias que faciliten al niño el desarrollo de esta habilidad desde las aulas de clase y con la ayuda del docente.

- Se pudo detectar que el 52\% (test) de los niños de tercer grado de la Escuela de Educación Básica "La Granja" no han desarrollado las habilidades lectoras, limitando así su rendimiento y no poder alcance un nivel satisfactorio dentro de 
esta habilidad, por tanto, es necesario enseñar a los niños el placer de la lectura mediante los nuevos recursos tecnológicos.

- Además, se establece la necesidad de valorar los recursos didácticos por método de especialistas de acuerdo a su criterio técnico, para mejoren las habilidades lectoras en los niños (as) de tercer grado de la Escuela de Educación Básica "la Granja”, enfocándose en las Tecnologías de Información y Comunicación TIC 'S en las cuales los niños de la nueva sociedad del conocimiento se encuentran inmersos, siendo así una herramienta educativa poderosa para el docente.

\section{Referencias.}

Alaís, A., \& Sarmiento, J. (2014). Mejoramiento de la comprensión lectora en los estudiantes de cuarto grado de básica primaria mediante el desarrollo de estrategias cognitivas con el apoyo de un Recurso Tic. Bogotá: Universidad de la Sabana.

Castillo, Y. (2017). Desarrollar la habilidad lectora en alumnos de primer grado de primaria. San Luis Potosí: Congreso Nacional de Investigación Educativa.

Elosúa, M. R. (2014). Habilidades lectoras y rendimiento académico en $3^{\circ}$ y $6^{\circ}$ de Primaria: aspectos evolutivos y educativos. Madrid.

Fierro, M. (julio-septiembre de 2011). El desarrollo conceptual de la ciencia cognitiva. Parte I. Revista Colombiana de Psiquiatría, 40(3), pp. 519-533.

Guitart, M., Dolya, G., \& Veraksa, N. (2011). Aplicaciones educativas de la teoría Vygotskiana. El programa "key to learning". Revista Electrónica "Actualidades Investigativas en Educación", 11(2), pp. 1-22.

Gusqui, N., \& Tixi, J. (2017). Estrategias metodológicas de comprensión lectora para el desarrollo de la inteligencia lingüística de los estudiantes de cuarto año de educación general básica, de la escuela “Boyacá” parroquia San Gerardo, cantón Guano, provincia de Chimborazo, año lecti. Riobamba: Universidad Nacional de Chimborazo.

Gutierrez, C., \& Salmeron, H. (17 de Abril de 2012). Estrategias de Comprensión Lectora: Enseñanza y Evaluación en Educación Primaria. Revista Currículum y formación del profesorado, 16(1).

Gutiérrez, P. P. (2013). Competencias tecnológicas del profesorado en las universidades españolas. Ministerio de Educación del Perú, 27.

Hernandez, R. M. (2017). Impacto de las TIC en la educación: Retos y Perspectivas. Perú: Universidad San Ignacio de Loyola, Lima. 
López, N., Valladares, A., \& otros. (2014). Enseñanza de estrategías para la comprención de textos expositivos con alumnos de sexto año de primaria. Revista Mexicana de Investigación Educativa, 19(63), PP. 1047-1068.

Muñoz, A., \& Ocaña, M. (enero-junio de 2017). Uso de estrategias metacognitivas para la comprensión textual. Cuadernos de Lingüística Hispánica, pp. 223-244.

Pascual, J. (18 de 10 de 2018). TEST DE COMPRENSIÓN LECTORA. Obtenido de http://www.juntadeandalucia.es/averroes/centrostic/41701419/helvia/aula/archivos/repositorio/0/18/html/Lenguatic/Italica/format os_variados/Comprensio_lectora/test_comprension1.htm

Pikara, G. (10 de Junio de 2014). Metodología de la investigación, Método Inductivo y Deductivo. Obtenido de https://es.slideshare.net/pikaragabriela/metodologa-dela-investigacin-35727551

Ríos, F. J., \& Cardona, A. V. (2016). Procesos de aprendizaje en niños de 6 a 10 años de edad con antecedente de nacimiento prematuro. Colombia: Revista Latinoamericana de Ciencias Sociales, Niñez y Juventud.

Rodríguez, J., \& Maldonado, J. (2017). Estudio del lenguaje desde la perspectiva sociocultural. Revista Ánfora de la Universidad Autónoma de Mánizales, 24(43), $17-38$.

Serna, J. (Enero-Julio de 2015). Propuesta didáctica para la comprensión crítica en la Universidad La Gran Colombia. Cuadernos de Lingüística Hispánic(25), pp. 165180.

Tierra, J. (2016). La comprensión lectora en el rendimiento académico de lengua y literatura en los niños de quinto año de educación básica paralelo "e" de la Unidad Educativa $n^{\circ} 6$ "combatientes del tapi" provincia del Chimborazo cantón Riobamba año lectivo 2015-2016. Riobamba: Universidad Nacional de Chimborazo.

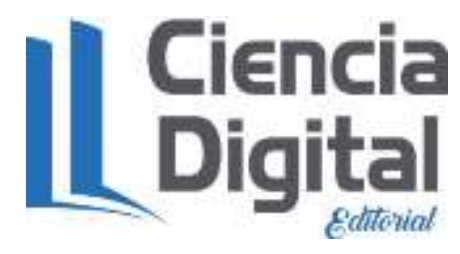

PARA CITAR EL ARTÍCULO INDEXADO. 
Pullupaxi Pullupaxi, P. del R., Navas Franco, L., Acosta Bonilla, J., \& Paredes Guevara, A. (2019). Los recursos didácticos tecnológicos en el desarrollo de las habilidades lectoras en los estudiantes de Educación Básica. Ciencia Digital, 3(2.6), 60-72. https://doi.org/10.33262/cienciadigital.v3i2.6.518

\section{\Ciencia}

El artículo que se publica es de exclusiva responsabilidad de los autores y no necesariamente reflejan el pensamiento de la Revista Ciencia Digital.

El artículo queda en propiedad de la revista y, por tanto, su publicación parcial y/o total en otro medio tiene que ser autorizado por el director de la Revista Ciencia Digital.
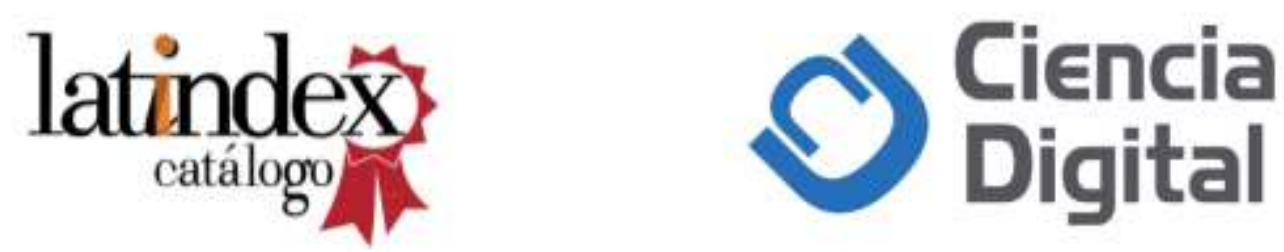